\title{
Letter to Editor: The Role of Wearable Technology in the Diagnosis and Prevention of COVID-19
}

\author{
Seyed Mojtaba Hosseini Bamakan" ${ }^{*}$ (D), Ehsan Rahbar $^{2}$ (D), Mehdi Gheisari ${ }^{3}$ (D)
}

1. Department of Industrial Management, Faculty of Humanities and Social Sciences, Yazd University, Yazd, Iran.

2. Department of Economics, Faculty of Management and Accounting, Yazd University, Yazd, Iran.

3. Department of Computer Science, Faculty of Engineering, Southern University of Technology and Science, Shenzhen, China.

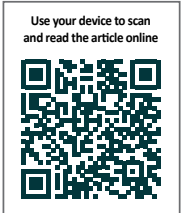

Citation Hosseini Bamakan SM, Rahbar E, Gheisari M. The Role of Wearable Technology in the Diagnosis and Prevention of COVID-19. Journal of Research \& Health. 2021; 11(4):213-214. http://dx.doi.org/10.32598/JRH.11.4.1903.1

http://dx.doi.org/10.32598/JRH.11.4.1903.1

\section{Dear Editor}

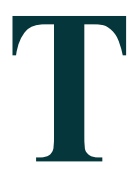

he Coronavirus Disease 2019(COVID-19) crisis, first detected in Wuhan, China, has dramatically affected healthcare systems, economies, political activities, and all aspects of our lives all over the world. The most well-known symptoms of COVID-19 are fever, dry cough, sore throat, and breathing difficulties. Nowadays, the role of technology innovations in precaution, early diagnosis, and treatment is not deniable due to the increase in various types of electronics. Some types of social media, such as Instagram are used by official organizations, like the World Health Organization (WHO) and UNICEF to raise public awareness [1]. According to the importance of eHealth, and the capability of Wearable Technology (WT) to integrate different facilities in one device, the use of WT is significant to enhance public health systems.

WT comprises a large group of worn gadgets, including watches, glasses, and bras, and is becoming a revolution assisting our daily life. Recently, this technology has been applied in domains, such as education, business, and the military. This is absolutely obvious that WT can be broadly used in the healthcare industry. In this correspondence, our main aim is to discuss the application of WTs in the health- care realm, their potentials to help us combat COVID-19, and its challenges.

A category of diagnostic wearable devices according to COVID-19 symptoms and body reactions is presented as follows: respiratory tracking devices, heart rate tracking devices, temperature tracking devices, breathing/cough monitoring devices, and disquiet/depression disorder treatment devices.

First, low levels of saturation of oxygen in the blood can be perilous to human health. Respiratory assessment approaches, such as transmission pulse oximetry and reflection pulse oximetry have been developed lately and used by respiratory therapists. A pulse oximeter known as a portable and wearable device is an arterial oxygen saturation test, which can be placed in different parts of the body.

Second, the heart rate change is considered to be a very important medical sign of diseases. Photoplethysmography (PPG) is an optical methodology that is used in wearables to detect and record heart rate in different environments almost uninterruptedly [2]. These wearables, sometimes with the ability of recording arrhythmia, could be a great success in detecting COVID-19 and tackling it in time.

\footnotetext{
* Corresponding Author:

Seyed Mojtaba Hosseini Bamakan, PhD.

Address: Department of Industrial Management, Faculty of Humanities and Social Sciences, Yazd University, Yazd, Iran. Phone: +98 (35) 31233751

E-mail: smhosseini@yazd.ac.ir
} 
Third, body temperature is a remarkably meaningful indicator of human health. Temp Traq ${ }^{\mathrm{TM}}$ patches take the core temperature of the body and send it to a smartphone [3]. Overall, making use of these temperature data is to assist us in providing care for COVID-19 patients.

Forth, trying to measure breathing patterns, for instance, when the person is in different environments, doing physical activities, and sleeping than in a stable condition is quite challenging. The Respiratory Inductance Plethysmography (RIP) method widely used in various types of medical wearable devices, is well-known as an accepted practice to measure pulmonary ventilation by tracking the movements of the abdomen and chest [4]. A very low-cost way to detect and monitor cough is to use a microphone worn on a surface near to airways. MobiCough using data from a microphone is a suitable representative of this category [5]. Cough monitoring wearable technology is assumed to have an important role in diagnosing COVID-19.

Finally, neuromodulation is a process of utilizing electrical impulses in the body, which is known as a treatment. Applying neuromodulators to cure anxiousness and depression disorders is considered to be impressively beneficial to suspected or confirmed COVID-19 cases. WT is a growing technology practiced by interdisciplinary fields to play a paramount role in treating COVID-19 [6].

Innovative technology has been a great assistant to health care services facing pandemic outbreaks simultaneously hitting different societies around the world. In the current situation and the number of cases with Ebola, Temporal Network Analysis was successfully applied in the medical procedures [7]. Having several competitive advantages, including portability, data transfer, and simplicity, WT as any other type of technology requires dealing with many challenges, such as battery durability, flexibility, clinical acceptance, and marketability. It is stated that wearable eye contact lenses could be a new promising tear-based technology for detecting COVID-19 infection in suspected cases as they were applied to evaluate the level of glucose in diabetics. Nevertheless, this proposal needs to pass different medical experiments to be validated. Undoubtedly, new technologies, such as WT will be more widely utilized in diagnosing and treatment of COVID-19.

\section{Ethical Considerations}

\section{Compliance with ethical guidelines}

There were no ethical considerations to be considered in this research.

\section{Funding}

This research did not receive any grant from funding agencies in the public, commercial, or non-profit sectors.

\section{Authors' contributions}

All authors equally contributed in preparing this article.

\section{Conflict of interest}

The authors declared no conflict of interest.

\section{References}

[1] Hosseini Bamakan SM, Haddadpoor Jahromi MJ. Role of social responsibility in prevention of the COVID-19 outbreak from systems thinking perspective. Public Health. 2021; 190:e18-20. [PMID] [DOI:10.1038/s41746-020-0297-4]

[2] Nelson BW, Low CA, Jacobson N, Areán P, Torous J, Allen NB. Guidelines for wrist-worn consumer wearable assessment of heart rate in biobehavioral research. NPJ Digit Med. 2020; 3:90. [DOI:10.1038/s41746-020-0297-4] [PMID]

[3] Tamura T, Huang M, Togawa T. Body temperature, heat flow, and evaporation. In: Tamura $\mathrm{T}$, Chen $\mathrm{T}$, editors. Seamless healthcare monitoring: Advancements in wearable, attachable, and invisible devices. New York: Springer International Publishing; 2017. pp. 281-307. https://www. google.com/books/edition/Seamless_Healthcare_Monitoring/ dERADwAAQBAJ?hl=en\&gbpv $=0$

[4] Elfaramawy T, Fall CL, Morissette M, Lellouche F, Gosselin B. Wireless respiratory monitoring and coughing detection using a wearable patch sensor network. Paper presented at: $15^{\text {th }}$ IEEE International New Circuits and Systems Conference (NEWCAS). 25-28 June 2017; Strasbourg, France. [DOI:10.1038/s41746-0200297-4]

[5] Pham C. MobiCough: Real-time cough detection and monitoring using low-cost mobile devices. In: Nguyen NT, Trawiński B, Fujita $\mathrm{H}$, Hong TP, editors. Intelligent information and database systems. ACIIDS 2016. Lecture notes in computer science. Berlin: Springer; 2016. [DOI:10.1038/s41746-020-0297-4]

[6] Shiraz, AN, Leaker B, Demosthenous A. Wearable neuromodulators. In: Ortiz JH, editor. Wearable technologies. London: IntechOpen; 2018. https://www.google.com/books/edition/ Wearable_Technologies/wHuQDwAAQBAJ?hl=en\&gbpv=0

[7] Sareen S, Sood SK, Gupta SK. IoT-based cloud framework to control Ebola virus outbreak. J Ambient Intell Humaniz Comput. 2018; 9(3):459-76. [DOI:10.1007/s12652-016-0427-7] 